\title{
Higher Chain Formula proved by Combinatorics
}

\author{
Tsoy-Wo Ma * \\ School of Mathematics and Statistics, \\ University of Western Australia, \\ Nedlands, W.A. , 6907, Australia. \\ twma@maths. uwa.edu. au
}

Submitted: Dec 5, 2008; Accepted: Jun 13, 2009; Published: Jun 19, 2009

Mathematics Subject Classifications: 05A05, 05A10, 05A17

\begin{abstract}
We present an elementary combinatorial proof of a formula to express the higher partial derivatives of composite functions in terms of those of factor functions.
\end{abstract}

1. Introduction. Consider $h: x \in X \subset \mathbb{R}^{\nu} \stackrel{f}{\longrightarrow} y \in Y \subset \mathbb{R}^{\mu} \stackrel{g}{\longrightarrow} z \in \mathbb{R}$ where $X, Y$ are open subsets of $\mathbb{R}^{\nu}, \mathbb{R}^{\mu}$ respectively and $f, g$ are sufficiently smooth functions. Lots of work have been done to express the higher partial derivatives of the composite function $h=g f$ in terms of those of $f, g$. Among many important contributions not included in our references, see [1], [2], [3] and [4] for $\mu=\nu=1$. See [5] for $\nu=1$ and any $\mu$. See [6] and [7] for $\mu=\nu=2$. Finally see [8], [9] [10], [11] and [12] for the general case. Here we propose a concise version in $\S 6$ with a combinatorial proof modified from [13] extending $\mu$ from their one dimension to our many dimensions. This paper is self-contained and should be readable by broad audiences including students studying routine operations of partial differentiation. In addition to many other applications, the corollary of this strategically important formula is required to investigate holomorphic functions on test spaces under coordinate transformations.

2. It is more intuitive to work with variables $x=\left(x_{1}, \cdots, x_{\nu}\right)$ and $y=\left(y_{1}, \cdots, y_{\mu}\right)$. For each $k$ in the set $J_{n}$ of integers $1,2, \cdots, n$, let $t_{k}$ denote one of the independent variables $x_{1}, \cdots, x_{\nu}$. A partition of $J_{n}$ is a family of pairwise disjoint nonempty subsets of $J_{n}$ whose union is $J_{n}$. Sets in a partition are called blocks. A block function is to assign a label to each block of a partition. The set of all functions from a partition $P$ of $J_{n}$ into $J_{\mu}$ is denoted by $P_{\mu}$. The set of all partitions of $J_{n}$ is denoted by $\mathbb{P}_{n}$.

3. Lemma.

$$
\frac{\partial^{n} z}{\partial t_{1} \cdots \partial t_{n}}=\sum_{P \in \mathbb{P}_{n}} \sum_{\lambda \in P_{\mu}}\left\{\left(\prod_{B \in P} \frac{\partial}{\partial y_{\lambda(B)}}\right) z\right\}\left\{\prod_{B \in P}\left[\left(\prod_{b \in B} \frac{\partial}{\partial t_{b}}\right) y_{\lambda(B)}\right]\right\} .
$$

*Dedicated to Galileo Galilei and Giordano Bruno 
In fact, it is obviously true for $n=1$. Inductively, differentiation with respect to $t_{n+1}$ produces terms that are products of factors of the form

$$
\left\{\left(\prod_{B \in P} \frac{\partial}{\partial y_{\lambda(B)}}\right) z\right\}\left\{\prod_{B \in P, B \neq A \in P}\left[\left(\prod_{b \in B} \frac{\partial}{\partial t_{b}}\right) y_{\lambda(B)}\right]\right\} \frac{\partial}{\partial t_{n+1}}\left(\prod_{a \in A} \frac{\partial}{\partial t_{a}}\right) y_{\lambda(A)}
$$

and

$$
\left\{\frac{\partial}{\partial t_{n+1}}\left(\prod_{B \in P} \frac{\partial}{\partial y_{\lambda(B)}}\right) z\right\}\left\{\prod_{B \in P}\left[\left(\prod_{b \in B} \frac{\partial}{\partial t_{b}}\right) y_{\lambda(B)}\right]\right\} .
$$

$\operatorname{Eq}(3.1)$ corresponds to a partition of $J_{n+1}$ obtained by adding $n+1$ to block $A$ of $P$ while all other blocks remain to be the same. We do not need to change any label of any block. For $\operatorname{Eq}(3.2)$, the family $Q=P \cup\left\{S_{n+1}\right\}$ is a partition of $J_{n+1}$ where $S_{n+1}=\{n+1\}$. Define a function $\varphi_{i}: Q \rightarrow J_{\mu}$ by $\varphi_{i}\left(S_{n+1}\right)=i$ and $\varphi_{i}(B)=\lambda(B)$ for all $B \in P$. Then $\operatorname{Eq}(3.2)$ becomes

$$
\begin{aligned}
& \left\{\sum_{i=1}^{\mu} \frac{\partial y_{i}}{\partial t_{n+1}} \frac{\partial}{\partial y_{i}}\left(\prod_{B \in P} \frac{\partial}{\partial y_{\lambda(B)}}\right) z\right\}\left\{\prod_{B \in P}\left[\left(\prod_{b \in B} \frac{\partial}{\partial t_{b}}\right) y_{\lambda(B)}\right]\right\} \\
= & \left\{\sum_{i=1}^{\mu} \frac{\partial}{\partial y_{i}}\left(\prod_{B \in P} \frac{\partial}{\partial y_{\lambda(B)}}\right) z\right\}\left\{\frac{\partial y_{i}}{\partial t_{n+1}} \prod_{B \in P}\left[\left(\prod_{b \in B} \frac{\partial}{\partial t_{b}}\right) y_{\lambda(B)}\right]\right\} \\
= & \sum_{i=1}^{\mu}\left\{\left(\prod_{A \in Q} \frac{\partial}{\partial y_{\varphi_{i}(A)}}\right) z\right\}\left\{\prod_{A \in Q}\left[\left(\prod_{a \in A} \frac{\partial}{\partial t_{a}}\right) y_{\varphi_{i}(A)}\right]\right\} .
\end{aligned}
$$

Clearly this covers all cases for $Q \in \mathbb{P}_{n+1}$ and $\varphi \in Q_{\mu}$. Hence we finish the proof.

4. For every multi-index $\alpha=\left(\alpha_{1}, \cdots, \alpha_{\nu}\right) \in \mathbb{N}^{\nu}$ of length $\nu$ where $\mathbb{N}$ is the set of all nonnegative integers and for every $x \in \mathbb{R}^{\nu}$, let

$$
|\alpha|=\sum_{j=1}^{\nu} \alpha_{j}, \quad \alpha !=\prod_{j=1}^{\nu} \alpha_{j} !, \quad x^{\alpha}=\prod_{j=1}^{\nu} x_{j}^{\alpha_{j}}, \quad \frac{\partial^{|\alpha|} z}{\partial x^{\alpha}}=\prod_{j=1}^{\nu}\left(\frac{\partial}{\partial x_{j}}\right)^{\alpha_{j}} z .
$$

By convention, define $\theta^{0}=1$ regardless whether $\theta$ is a number or a differential operator. Suppose that $\left\{e_{j}: j \in J_{\mu}\right\}$ denotes the standard basis of $\mathbb{R}^{\mu}$. In particular each $e_{j}$ is a multi-index of length $\mu$.

5. A multi-index $\alpha$ in $\mathbb{R}^{\nu}$ is said to be decomposed into $s$ parts $p_{1}, \cdots, p_{s}$ in $\mathbb{N}^{\nu}$ with multiplicities $m_{1}, \cdots, m_{s}$ in $\mathbb{N}^{\mu}$ respectively if the decomposition equation

$$
\alpha=\left|m_{1}\right| p_{1}+\left|m_{2}\right| p_{2}+\cdots+\left|m_{s}\right| p_{s}
$$

holds and all parts are different. Note that the parts $p$ 's and the multiplicities $m$ 's are multi-indexes of order $\nu, \mu$ respectively. In this case, the total multiplicity is defined by

$$
m=m_{1}+m_{2}+\cdots+m_{s} .
$$


The list $(s, p, m)$ is called a $\mu$-decomposition or just a decomposition of $\alpha$. One of many ways to ensure that the parts are all different is to define $\alpha \ll \beta$ if there is $j \in J_{\nu}$ such that $\alpha_{1}=\beta_{1}, \cdots, \alpha_{j-1}=\beta_{j-1}$ but $\alpha_{j}<\beta_{j}$. We may demand the parts of a decomposition $(s, p, m)$ to satisfy the additional condition $0 \ll p_{1} \ll p_{2} \ll \cdots \ll p_{s}$.

\section{Higher Chain Formula.}

$$
\frac{\partial^{|\alpha|} z}{\partial x^{\alpha}}=\alpha ! \sum_{(s, p, m) \in \mathscr{D}} \frac{\partial^{|m|} z}{\partial y^{m}} \prod_{k=1}^{s} \frac{1}{m_{k} !}\left[\frac{1}{p_{k} !} \frac{\partial^{\left|p_{k}\right|} y}{\partial x^{p_{k}}}\right]^{m_{k}}
$$

where $\mathscr{D}$ is the set of all decompositions of $\alpha$. Let $p_{k}=\left(p_{k 1}, \cdots, p_{k \nu}\right), m_{k}=\left(m_{k 1}, \cdots, m_{k \mu}\right)$ and $r_{i}=m_{1 i}+\cdots+m_{s i}$. Clearly we have $m=\left(r_{1}, \cdots, r_{\mu}\right)$. The terms on the right hand side in long form become

$$
\alpha ! \frac{\partial^{r_{1}+\cdots+r_{\mu}} z}{\partial y_{1}^{r_{1}} \cdots \partial y_{\mu}^{r_{\mu}}} \prod_{k=1}^{s} \prod_{i=1}^{\mu} \frac{1}{m_{k i} !}\left[\frac{1}{p_{k 1} ! \cdots p_{k \nu} !} \frac{\partial^{p_{k 1}+\cdots+p_{k \nu}} y_{i}}{\partial x^{p_{k 1} \cdots \partial x^{p_{k \nu}}}}\right]^{m_{k i}}
$$

7. We shall prove this formula by interpretation rather than by formal argument. Let us examine the special case when $\nu=4, \mu=2$ and $\alpha=(39,50,9,0)$. We have independent variables $x_{1}, x_{2}, x_{3}, x_{4}$ and dependent variables $y_{1}, y_{2}$. For

$$
\begin{aligned}
t_{1} & =\cdots=t_{39}=x_{1} \\
t_{40} & =\cdots=t_{89}=x_{2} \\
t_{90} & =\cdots=t_{98}=x_{3}
\end{aligned}
$$

the left hand side of the lemma becomes

$$
\frac{\partial^{|\alpha|} z}{\partial x^{\alpha}}=\frac{\partial^{98} z}{\partial x_{1}^{39} \partial x_{2}^{50} \partial x_{3}^{9}}=\frac{\partial^{98} z}{\partial t_{1} \cdots \partial t_{98}} .
$$

8. Consider a partition $P$ of $J_{98}$ whose first 5 blocks are displayed by the following table:

\begin{tabular}{|c|c|c|c|c|c|c|}
\hline $\mathrm{A}$ & $\mathrm{B}$ & $\mathrm{C}$ & $\mathrm{D}$ & $\mathrm{E}$ & $\mathrm{F}$ & $\mathrm{G}$ \\
\hline 1 & 2 & $e_{2}$ & $1,2,3,4$ & $x_{1}, x_{1}, x_{1}, x_{1}$ & $40,41,42,43,44$ & $x_{2}, x_{2}, x_{2}, x_{2}, x_{2}$ \\
2 & 1 & $e_{1}$ & $5,6,7,8$ & $x_{1}, x_{1}, x_{1}, x_{1}$ & $45,46,47,48,49$ & $x_{2}, x_{2}, x_{2}, x_{2}, x_{2}$ \\
3 & 1 & $e_{1}$ & $9,10,11,12$ & $x_{1}, x_{1}, x_{1}, x_{1}$ & $50,51,52,63,54$ & $x_{2}, x_{2}, x_{2}, x_{2}, x_{2}$ \\
4 & 2 & $e_{2}$ & $13,14,15,16$ & $x_{1}, x_{1}, x_{1}, x_{1}$ & $55,56,57,58,59$ & $x_{2}, x_{2}, x_{2}, x_{2}, x_{2}$ \\
5 & 2 & $e_{2}$ & $17,18,19,20$ & $x_{1}, x_{1}, x_{1}, x_{1}$ & $60,61,62,63,64$ & $x_{2}, x_{2}, x_{2}, x_{2}, x_{2}$ \\
\hline
\end{tabular}

Column-A is the serial number of the blocks. Column-B assigns an integer label to each block in order to define the block function $\lambda$, for example $\lambda_{3}=1$. Column-C uses the basis of $\mathbb{R}^{\mu}$ to label the blocks. Hence the first multiplicity is $m_{1}=2 e_{1}+3 e_{2}=(2,3)$. Column-D indicates the integers in each block that produce $4 x_{1}$ in column-E. Column-F indicates the integers in each block that produce $5 x_{2}$ in column-G. Since no $x_{3}, x_{4}$ are 
involved, the first part is $p_{1}=(4,5,0,0)$. In this particular example, the other blocks of $P$ and corresponding parts with multiplicities are

\begin{tabular}{|c|c|c|c|c|c|c|c|c|}
\hline $\mathrm{A} 2$ & $\mathrm{~B} 2$ & $\mathrm{C} 2$ & $\mathrm{D} 2$ & $\mathrm{E} 2$ & $\mathrm{~F} 2$ & $\mathrm{G} 2$ & $\mathrm{H} 2$ & $\mathrm{~J} 2$ \\
\hline 7 & 1 & $e_{1}$ & 21,22 & $x_{1}, x_{1}$ & $65,66,67$ & $x_{2}, x_{2}, x_{2}$ & 90 & $x_{3}$ \\
$\ldots$ & $\ldots$ & $\ldots$ & $\ldots$ & $\ldots$ & $\ldots$ & $\ldots$ & $\ldots$ & $\ldots$ \\
13 & 2 & $e_{2}$ & 33,34 & $x_{1}, x_{1}$ & $83,84,85$ & $x_{2}, x_{2}, x_{2}$ & 96 & $x_{3}$ \\
\hline
\end{tabular}

and

\begin{tabular}{|c|c|c|c|c|c|c|c|c|}
\hline A3 & B3 & C3 & D3 & E3 & F3 & G3 & H3 & J3 \\
\hline 14 & 2 & $e_{2}$ & $35, \cdots, 39$ & $x_{1}, \cdots, x_{1}$ & $56, \cdots, 59$ & $x_{2}, \cdots, x_{2}$ & 97,98 & $x_{3}, x_{3}$ \\
\hline
\end{tabular}

This partition can be compactly represented by the decomposition equation

$$
(39,50,9,0)=|(2,3)|(4,5,0,0)+|(3,4)|(2,3,1,0)+|(0,1)|(5,4,2,0) .
$$

We set $m_{2}=(3,4), p_{2}=(2,3,1,0), m_{3}=(0,1)$ and $p_{3}=(5,4,2,0)$. The total multiplicity is $m=m_{1}+m_{2}+m_{3}=(5,8)$.

9. The block function restricted to column-B offers the differential operator

$$
\frac{\partial}{\partial y_{2}} \frac{\partial}{\partial y_{1}} \frac{\partial}{\partial y_{1}} \frac{\partial}{\partial y_{2}} \frac{\partial}{\partial y_{2}}=\left(\frac{\partial}{\partial y_{1}}\right)^{2}\left(\frac{\partial}{\partial y_{2}}\right)^{3}
$$

Three tables together give

$$
\left(\prod_{B \in P} \frac{\partial}{\partial y_{\lambda(B)}}\right) z=\left(\frac{\partial}{\partial y_{1}}\right)^{2+3+0}\left(\frac{\partial}{\partial y_{2}}\right)^{3+4+1} z=\frac{\partial^{13} z}{\partial y_{1}^{5} \partial x_{2}^{8}}=\frac{\partial^{|m|} z}{\partial y^{m}}
$$

Next for the first block in table-1, we obtain

$$
\left(\prod_{b \in B} \frac{\partial}{\partial t_{b}}\right) y_{\lambda(B)}=\left(\frac{\partial}{\partial x_{1}} \frac{\partial}{\partial x_{1}} \frac{\partial}{\partial x_{1}} \frac{\partial}{\partial x_{1}}\right)\left(\frac{\partial}{\partial x_{2}} \frac{\partial}{\partial x_{2}} \frac{\partial}{\partial x_{2}} \frac{\partial}{\partial x_{2}} \frac{\partial}{\partial x_{2}}\right) y_{2} .
$$

All rows in three tables together produce

$$
\begin{aligned}
& \prod_{B \in P}\left[\left(\prod_{b \in B} \frac{\partial}{\partial x_{b}}\right) y_{\lambda(B)}\right] \\
&=\left(\frac{\partial^{9} y_{1}}{\partial x_{1}^{4} \partial x_{2}^{5}}\right)^{2}\left(\frac{\partial^{9} y_{2}}{\partial x_{1}^{4} \partial x_{2}^{5}}\right)^{3} \cdot\left(\frac{\partial^{6} y_{1}}{\partial x_{1}^{2} \partial x_{2}^{3} \partial x_{3}}\right)^{3}\left(\frac{\partial^{6} y_{2}}{\partial x_{1}^{2} \partial x_{2}^{3} \partial x_{3}}\right)^{4} \\
& \cdot\left(\frac{\partial^{17} y_{1}}{\partial x_{1}^{5} \partial x_{2}^{4} \partial x_{3}^{2}}\right)^{0}\left(\frac{\partial^{17} y_{2}}{\partial x_{1}^{5} \partial x_{2}^{4} \partial x_{3}^{2}}\right)^{1} \\
&= {\left[\frac{\partial^{\left|p_{1}\right|} y}{\partial x^{p_{1}}}\right]^{m_{1}}\left[\frac{\partial^{\left|p_{2}\right|} y}{\partial x^{p_{2}}}\right]^{m_{2}}\left[\frac{\partial^{\left|p_{3}\right|} y}{\partial x^{p_{3}}}\right]^{m_{3}}=\prod_{k=1}^{s}\left[\frac{\partial^{\left|p_{k}\right|} y}{\partial x^{p_{k}}}\right]^{m_{k}} . }
\end{aligned}
$$


This is simplified to

$$
\frac{\partial^{13} z}{\partial y_{1}^{5} \partial x_{2}^{8}}\left(\frac{\partial^{9} y_{1}}{\partial x_{1}^{4} \partial x_{2}^{5}}\right)^{2}\left(\frac{\partial^{9} y_{2}}{\partial x_{1}^{4} \partial x_{2}^{5}}\right)^{3}\left(\frac{\partial^{6} y_{1}}{\partial x_{1}^{2} \partial x_{2}^{3} \partial x_{3}}\right)^{3}\left(\frac{\partial^{6} y_{2}}{\partial x_{1}^{2} \partial x_{2}^{3} \partial x_{3}}\right)^{4}\left(\frac{\partial^{17} y_{2}}{\partial x_{1}^{5} \partial x_{2}^{4} \partial x_{3}^{2}}\right) \text {. }
$$

10. The columns E, E2 and E3 remain to be the same if we permute the order of integers in each cell of columns D, D2 and D3. Hence the number of different ways to distribute the integers $1,2, \cdots, 39$ into columns D, D2 and D3 is $39 ! /\left(4 !^{5} 2 !^{7} 5 !\right)$. Taking all variables $x_{1}, \cdots, x_{\mu}$ into account, we have

$$
\frac{39 !}{4 !^{5} 2 !^{7} 5 !} \frac{50 !}{5^{5} 3 !^{7} 4 !} \frac{9 !}{0 !^{5} 1 !^{7} 2 !} .
$$

If we permute the rows of each table, it does not alter the columns E, G, E2, G2, J2, E3, G3 and J3. Hence the total number of different ways to distribute all integers in $J_{98}$ is

$$
\left(\frac{39 !}{4 !^{5} 2 !^{7} 5 !} \frac{50 !}{5^{5} 3 !^{7} 4 !} \frac{9 !}{0 !^{5} 1 !^{7} 2 !}\right)\left(\frac{1}{5 ! 7 ! 1 !}\right)=\left(\prod_{j=1}^{\nu} \frac{\alpha_{j} !}{\prod_{k=1}^{s}\left(p_{k j} !\right)^{\left|m_{k}\right|}}\right) \frac{1}{\prod_{k=1}^{s}\left|m_{k}\right| !}
$$

On the other hand if we interchange rows 2,3 without altering their serial numbers in table-1, we have different block functions with the same effect on the term (9.1). Hence the number of different ways to distribute the integers $1,2,3,4,5$ is $5 ! /(2$ ! 3 !). Because we demand that the parts are all different, the total number of different block functions is

$$
\frac{5 !}{2 ! 3 !} \frac{7 !}{3 ! 4 !} \frac{1 !}{0 ! 1 !}=\prod_{k=1}^{s} \frac{\left|m_{k}\right| !}{\prod_{i=1}^{\mu} m_{k i} !} .
$$

Putting everything together, we obtain

$$
\begin{aligned}
& \left\{\left(\prod_{j=1}^{\nu} \frac{\alpha_{j} !}{\prod_{k=1}^{s}\left(p_{k j} !\right)^{\left|m_{k}\right|}}\right) \frac{1}{\prod_{k=1}^{s}\left|m_{k}\right| !}\right\}\left(\prod_{k=1}^{s} \frac{\left|m_{k}\right| !}{\prod_{i=1}^{\mu} m_{k i} !}\right) \\
= & \left(\alpha ! \prod_{j=1}^{\nu} \prod_{k=1}^{s} \frac{1}{\left(p_{k j} !\right)^{m_{k 1}+\cdots+m_{k \mu}}}\right)\left(\prod_{k=1}^{s} \prod_{i=1}^{\mu} \frac{1}{m_{k i} !}\right) \\
= & \alpha !\left(\prod_{j=1}^{\nu} \prod_{k=1}^{s} \prod_{i=1}^{\mu} \frac{1}{\left(p_{k j} !\right)^{m_{k i}}}\right)\left(\prod_{k=1}^{s} \prod_{i=1}^{\mu} \frac{1}{m_{k i} !}\right) \\
= & \alpha ! \prod_{k=1}^{s} \prod_{i=1}^{\mu} \frac{1}{m_{k i} ! \prod_{j=1}^{\nu}\left(p_{k j} !\right)^{m_{k i}}}
\end{aligned}
$$

which is identical to the coefficient of the term (6.1). This completes the proof.

11. Consider an alternative illustrative example to find all possible decompositions. Suppose $\mu=2, \nu=3$ and $\alpha=(3,7,5)$. The maximum among $\alpha_{1}, \alpha_{2}, \alpha_{3}$ is 7 . Solve for nonnegative integers $\alpha_{i j}$ from the traditional equations

$$
\alpha_{i}=\alpha_{i 1}+2 \alpha_{i 2}+3 \alpha_{i 3}+4 \alpha_{i 4}+5 \alpha_{i 5}+6 \alpha_{i 6}+7 \alpha_{i 7}, \quad \text { for } i=1,2,3 .
$$


One of many solutions is

$$
\left\{\begin{array}{l}
3=1+2 \cdot 1+3 \cdot 0+4 \cdot 0+5 \cdot 0+6 \cdot 0+7 \cdot 0 \\
7=1+2 \cdot 1+3 \cdot 0+4 \cdot 1+5 \cdot 0+6 \cdot 0+7 \cdot 0 \\
5=0+2 \cdot 0+3 \cdot 0+4 \cdot 0+5 \cdot 1+6 \cdot 0+7 \cdot 0
\end{array}\right.
$$

The nonzero columns are $(1,1,0),(1,1,0),(0,1,0)$ and $(0,0,1)$. Hence we get an equation

$$
(3,7,5)=(1,1,0)+2(1,1,0)+4(0,1,0)+5(0,0,1) .
$$

Combine the first two terms so that all parts are different,

$$
(3,7,5)=3(1,1,0)+4(0,1,0)+5(0,0,1) .
$$

Arrange the solutions into $0 \ll p_{1} \ll p_{2} \ll p_{3}$ where $p_{1}=(0,0,1), p_{2}=(0,1,0)$ and $p_{3}=(1,1,0)$ so that $\left|m_{1}\right|=5,\left|m_{2}\right|=4$ and $\left|m_{3}\right|=3$. One of many possible cases is $m_{1}=(3,2), m_{2}=(0,4)$ and $m_{3}=(2,1)$. In this way, we obtain a decomposition $\alpha=\left|m_{1}\right| p_{1}+\left|m_{2}\right| p_{2}+\left|m_{3}\right| p_{3}$.

12. Corollary. For $n=|\alpha|, m \in \mathbb{N}^{\mu}$ and $\beta \in \mathbb{N}^{\nu}$, we have

$$
\left|\frac{\partial^{|\alpha|} z}{\partial x^{\alpha}}\right| \leq(1+n)^{\mu+\nu+n} A(1+B)^{n}
$$

where

$$
A=\max _{|m| \leq n}\left|\frac{\partial^{|m|} z}{\partial y^{m}}\right| \quad \text { and } \quad B=\max _{1 \leq i \leq \mu} \max _{|\beta| \leq n}\left|\frac{\partial^{|\beta|} y_{i}}{\partial x^{\beta}}\right| .
$$

13. Indeed, from $\sum_{k=1}^{s}\left|m_{k}\right| p_{k j}=\alpha_{j}$, we obtain

$$
\sum_{k=1}^{s}\left|m_{k}\right|\left|p_{k}\right|=\sum_{k=1}^{s}\left|m_{k}\right| \sum_{j=1}^{\nu} p_{k j}=\sum_{j=1}^{\nu} \sum_{k=1}^{s}\left|m_{k}\right| p_{k j}=\sum_{j=1}^{\nu} \alpha_{j}=|\alpha|=n .
$$

In particular, we get $s \leq n,\left|m_{k}\right| \leq n,\left|p_{k}\right| \leq n$ and

$$
|m|=\sum_{i=1}^{\mu} r_{i}=\sum_{i=1}^{\mu} \sum_{k=1}^{s} m_{k i}=\sum_{k=1}^{s} \sum_{i=1}^{\mu} m_{k i}=\sum_{k=1}^{s}\left|m_{k}\right| \leq \sum_{k=1}^{s}\left|m_{k}\right|\left|p_{k}\right|=n .
$$

Furthermore, we get

$$
\begin{aligned}
\left|\frac{\partial^{|\alpha|} z}{\partial x^{\alpha}}\right| & =\left|\alpha ! \sum_{(s, p, m) \in \mathscr{D}} \frac{\partial^{|m|} z}{\partial y^{m}} \prod_{k=1}^{s} \frac{1}{m_{k} !}\left[\frac{1}{p_{k} !} \frac{\partial^{\left|p_{k}\right|} y}{\partial x^{p_{k}}}\right]^{m_{k}}\right| \\
& \leq \alpha ! \sum_{(s, p, m) \in \mathscr{D}}\left|\frac{\partial^{|m|} z}{\partial y^{m}}\right|\left|\prod_{k=1}^{s}\left[\frac{\partial^{\left|p_{k}\right|} y}{\partial x^{p_{k}}}\right]^{m_{k}}\right|=n ! \sum_{(s, p, m) \in \mathscr{D}} A \prod_{k=1}^{s} \prod_{i=1}^{\mu}\left|\frac{\partial^{\left|p_{k}\right|} y_{i}}{\partial x^{p_{k}}}\right|^{m_{k i}} \\
& \leq n ! \sum_{(s, p, m) \in \mathscr{D}} A \prod_{k=1}^{s} \prod_{i=1}^{\mu} B^{m_{k i}}=n ! \sum_{(s, p, m) \in \mathscr{D}} A B^{\sum_{k=1}^{s} \sum_{i=1}^{\mu} m_{k i}} \\
& \leq n ! \sum_{(s, p, m) \in \mathscr{D}} A B^{|m|} \leq n ! \sum_{(s, p, m) \in \mathscr{D}} A(1+B)^{n} .
\end{aligned}
$$


For $m_{k}=\left(m_{k 1}, \cdots, m_{k \mu}\right) \in \mathbb{N}^{\mu}$ satisfying $\left|m_{k}\right| \leq n$, there are $n+1$ possibilities for each $m_{k i}$ from 0 to $n$. The totality of $m_{k}$ is no more than $(1+n)^{\mu}$. Similarly the totality of $p_{k} \in \mathbb{N}^{\nu}$ satisfying $\left|p_{k}\right| \leq n$ is no more than $(1+n)^{\nu}$. Hence the total number of elements in $\mathscr{D}$ is no more than $n(1+n)^{\mu+\nu}$. The result follows from $n ! n(1+n)^{\mu+\nu} \leq(1+n)^{\mu+\nu+n}$.

14. Acknowledgements. Thanks to Western Australia Liver Transplant Unit, its supporting infra-structures and the donor so that I have the second life to carry out my research.

\section{References}

[1] A. Dresden, The derivatives of composite functions. Amer. Math. Monthly 50, (1943). $9-12$.

[2] W.P. Johnson, The curious history of Faa di Bruno's Formula, Amer. Math. Monthly, 109 (2002), 217-227.

[3] K. Spindler, A short proof of the formula of Faa di Bruno. Elem. Math. 60 (2005), no. $1,33-35$.

[4] A. Craik, Prehistory of Faa di Bruno's Formula, Amer. Math. Monthly, 112 (2) 2005, 119-130.

[5] R.L. Mishkov, Generalization of the formula of Faa Di Bruno for a composite function with a vector argument, Internat. J. Math. and Math. Sci. 24 (2000), 481-491.

[6] B. S. Song, The general formula for higher-order derivatives of composite functions. (Chinese) Pure Appl. Math. (Xi'an) 5 (1989), 83-85.

[7] R.B. Leipnik and C.E.M. Pearce, The multivariate Faa di Bruno formula and multivariate Taylor expansions with explicit integral remainder term. ANZIAM J. 48 (2007), no. 3, 327-341.

[8] L.E. Fraenkel, Formulae for high derivatives of composite functions, Math. Proc. Cam. Phil. Soc. 83 (1978), 159-165.

[9] Z.Y. Shen, Calculation of high order derivatives of composite functions by a graphtheoretic method. (Chinese) Neimenggu Daxue Xuebao 16 (1985), no. 2, 175-182.

[10] H. Gzyl, Multidimensional extension of Faa di Bruno's formula. J. Math. Anal. Appl. 116 (1986), no. 2, 450-455.

[11] G.M. Constantine and T.H. Savits, A multivariate Faa di Bruno formula with applications. Trans. Amer. Math. Soc. 348 (1996), no. 2, 503-520.

[12] L. Hernadez Encinas and J. Munoz Masque, A short proof of the generalized Faa di Bruno's formula. Appl. Math. Lett. 16 (2003), no. 6, 975-979.

[13] M. Hardy, Combinatorics of partial derivatives, Electron. J. Combin. 13 (2006), \#R1. 